\title{
Convex Sets and Convex Combinations on Complex Linear Spaces
}

\author{
Hidenori Matsuzaki \\ Shinshu University \\ Nagano, Japan
}

\author{
Noboru Endou \\ Gifu National College of Technology \\ Japan
}

Yasunari Shidama

Shinshu University

Nagano, Japan

Summary. In this article, convex sets, convex combinations and convex hulls on complex linear spaces are introduced.

MML identifier: $\underline{\text { CONVEX4 }}$, version: $\underline{7.8 .10 \quad 4.99 .1005}$

The articles [19], [18], [9], [23], [24], [6], [25], [7], [20], [3], [22], [17], [2], [11], [8], [1], [5], [10], [14], [15], [4], [16], [21], [12], and [13] provide the terminology and notation for this paper.

\section{Complex Linear Combinations}

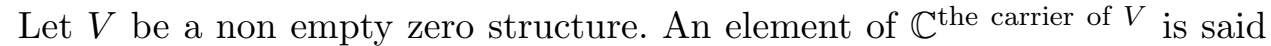
to be a $\mathbb{C}$-linear combination of $V$ if:

(Def. 1) There exists a finite subset $T$ of $V$ such that for every element $v$ of $V$ such that $v \notin T$ holds it $(v)=0$.

Let $V$ be a non empty additive loop structure and let $L$ be an element of

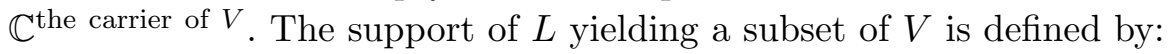

(Def. 2) The support of $L=\left\{v \in V: L(v) \neq 0_{\mathbb{C}}\right\}$.

Let $V$ be a non empty additive loop structure and let $L$ be a $\mathbb{C}$-linear combination of $V$. One can check that the support of $L$ is finite.

The following proposition is true 
(1) Let $V$ be a non empty additive loop structure, $L$ be a $\mathbb{C}$-linear combination of $V$, and $v$ be an element of $V$. Then $L(v)=0_{\mathbb{C}}$ if and only if $v \notin$ the support of $L$.

Let $V$ be a non empty additive loop structure. The functor ZeroCLC $V$ yields a $\mathbb{C}$-linear combination of $V$ and is defined by:

(Def. 3) The support of ZeroCLC $V=\emptyset$.

Let $V$ be a non empty additive loop structure. Note that the support of ZeroCLC $V$ is empty.

We now state the proposition

(2) For every non empty additive loop structure $V$ and for every element $v$ of $V$ holds (ZeroCLC $V)(v)=0_{\mathbb{C}}$.

Let $V$ be a non empty additive loop structure and let $A$ be a subset of $V$.

A $\mathbb{C}$-linear combination of $V$ is said to be a $\mathbb{C}$-linear combination of $A$ if:

(Def. 4) The support of it $\subseteq A$.

Next we state three propositions:

(3) Let $V$ be a non empty additive loop structure, $A, B$ be subsets of $V$, and $l$ be a $\mathbb{C}$-linear combination of $A$. If $A \subseteq B$, then $l$ is a $\mathbb{C}$-linear combination of $B$.

(4) Let $V$ be a non empty additive loop structure and $A$ be a subset of $V$. Then ZeroCLC $V$ is a $\mathbb{C}$-linear combination of $A$.

(5) Let $V$ be a non empty additive loop structure and $l$ be a $\mathbb{C}$-linear com-

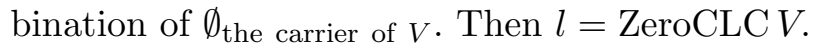

In the sequel $i$ is a natural number.

Let $V$ be a non empty CLS structure, let $F$ be a finite sequence of elements of the carrier of $V$, and let $f$ be a function from the carrier of $V$ into $\mathbb{C}$. The functor $f F$ yields a finite sequence of elements of the carrier of $V$ and is defined as follows:

(Def. 5) $\operatorname{len}(f F)=\operatorname{len} F$ and for every $i$ such that $i \in \operatorname{dom}(f F)$ holds $(f F)(i)=$ $f\left(F_{i}\right) \cdot F_{i}$.

For simplicity, we follow the rules: $V$ denotes a non empty CLS structure, $v, v_{1}, v_{2}, v_{3}$ denote vectors of $V, A$ denotes a subset of $V, l$ denotes a $\mathbb{C}$-linear combination of $A, x$ denotes a set, $a, b$ denote complex numbers, $F$ denotes a finite sequence of elements of the carrier of $V$, and $f$ denotes a function from the carrier of $V$ into $\mathbb{C}$.

The following propositions are true:

(6) If $x \in \operatorname{dom} F$ and $v=F(x)$, then $(f F)(x)=f(v) \cdot v$.

(7) $f \varepsilon_{(\text {the carrier of } V)}=\varepsilon_{(\text {the carrier of } V)}$.

(8) $f\langle v\rangle=\langle f(v) \cdot v\rangle$.

(9) $f\left\langle v_{1}, v_{2}\right\rangle=\left\langle f\left(v_{1}\right) \cdot v_{1}, f\left(v_{2}\right) \cdot v_{2}\right\rangle$. 
(10) $f\left\langle v_{1}, v_{2}, v_{3}\right\rangle=\left\langle f\left(v_{1}\right) \cdot v_{1}, f\left(v_{2}\right) \cdot v_{2}, f\left(v_{3}\right) \cdot v_{3}\right\rangle$.

In the sequel $L, L_{1}, L_{2}, L_{3}$ are $\mathbb{C}$-linear combinations of $V$.

Let $V$ be an Abelian add-associative right zeroed right complementable non empty CLS structure and let $L$ be a $\mathbb{C}$-linear combination of $V$. The functor $\sum L$ yields an element of $V$ and is defined by the condition (Def. 6).

(Def. 6) There exists a finite sequence $F$ of elements of the carrier of $V$ such that $F$ is one-to-one and $\operatorname{rng} F=$ the support of $L$ and $\sum L=\sum L F$.

One can prove the following propositions:

(11) For every Abelian add-associative right zeroed right complementable non empty CLS structure $V$ holds $\sum$ ZeroCLC $V=0_{V}$.

(12) Let $V$ be a complex linear space and $A$ be a subset of $V$. Suppose $A \neq \emptyset$. Then $A$ is linearly closed if and only if for every $\mathbb{C}$-linear combination $l$ of $A$ holds $\sum l \in A$.

(13) Let $V$ be an Abelian add-associative right zeroed right complementable non empty CLS structure and $l$ be a $\mathbb{C}$-linear combination of

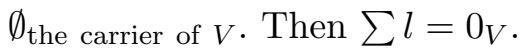

(14) Let $V$ be a complex linear space, $v$ be a vector of $V$, and $l$ be a $\mathbb{C}$-linear combination of $\{v\}$. Then $\sum l=l(v) \cdot v$.

(15) Let $V$ be a complex linear space and $v_{1}, v_{2}$ be vectors of $V$. Suppose $v_{1} \neq v_{2}$. Let $l$ be a $\mathbb{C}$-linear combination of $\left\{v_{1}, v_{2}\right\}$. Then $\sum l=l\left(v_{1}\right)$. $v_{1}+l\left(v_{2}\right) \cdot v_{2}$.

(16) Let $V$ be an Abelian add-associative right zeroed right complementable non empty CLS structure and $L$ be a $\mathbb{C}$-linear combination of $V$. If the support of $L=\emptyset$, then $\sum L=0_{V}$.

(17) Let $V$ be a complex linear space, $L$ be a $\mathbb{C}$-linear combination of $V$, and $v$ be a vector of $V$. If the support of $L=\{v\}$, then $\sum L=L(v) \cdot v$.

(18) Let $V$ be a complex linear space, $L$ be a $\mathbb{C}$-linear combination of $V$, and $v_{1}, v_{2}$ be vectors of $V$. If the support of $L=\left\{v_{1}, v_{2}\right\}$ and $v_{1} \neq v_{2}$, then $\sum L=L\left(v_{1}\right) \cdot v_{1}+L\left(v_{2}\right) \cdot v_{2}$.

Let $V$ be a non empty additive loop structure and let $L_{1}, L_{2}$ be $\mathbb{C}$-linear combinations of $V$. Let us observe that $L_{1}=L_{2}$ if and only if:

(Def. 7) For every element $v$ of $V$ holds $L_{1}(v)=L_{2}(v)$.

Let $V$ be a non empty additive loop structure and let $L_{1}, L_{2}$ be $\mathbb{C}$-linear combinations of $V$. Then $L_{1}+L_{2}$ is a $\mathbb{C}$-linear combination of $V$ and it can be characterized by the condition:

(Def. 8) For every element $v$ of $V$ holds $\left(L_{1}+L_{2}\right)(v)=L_{1}(v)+L_{2}(v)$.

One can prove the following propositions:

(19) The support of $L_{1}+L_{2} \subseteq$ (the support of $\left.L_{1}\right) \cup\left(\right.$ the support of $L_{2}$ ). 
(20) Suppose $L_{1}$ is a $\mathbb{C}$-linear combination of $A$ and $L_{2}$ is a $\mathbb{C}$-linear combination of $A$. Then $L_{1}+L_{2}$ is a $\mathbb{C}$-linear combination of $A$.

Let us consider $V, A$ and let $L_{1}, L_{2}$ be $\mathbb{C}$-linear combinations of $A$. Then $L_{1}+L_{2}$ is a $\mathbb{C}$-linear combination of $A$.

The following three propositions are true:

(21) For every non empty additive loop structure $V$ and for all $\mathbb{C}$-linear combinations $L_{1}, L_{2}$ of $V$ holds $L_{1}+L_{2}=L_{2}+L_{1}$.

(22) $L_{1}+\left(L_{2}+L_{3}\right)=\left(L_{1}+L_{2}\right)+L_{3}$.

(23) $L+$ ZeroCLC $V=L$.

Let us consider $V, a$ and let us consider $L$. The functor $a \cdot L$ yielding a $\mathbb{C}$-linear combination of $V$ is defined as follows:

(Def. 9) For every $v$ holds $(a \cdot L)(v)=a \cdot L(v)$.

One can prove the following propositions:

(24) If $a \neq 0_{\mathbb{C}}$, then the support of $a \cdot L=$ the support of $L$.

(25) $0_{\mathbb{C}} \cdot L=$ ZeroCLC $V$.

(26) If $L$ is a $\mathbb{C}$-linear combination of $A$, then $a \cdot L$ is a $\mathbb{C}$-linear combination of $A$.

(27) $(a+b) \cdot L=a \cdot L+b \cdot L$.

(28) $a \cdot\left(L_{1}+L_{2}\right)=a \cdot L_{1}+a \cdot L_{2}$.

(29) $a \cdot(b \cdot L)=(a \cdot b) \cdot L$.

(30) $1_{\mathbb{C}} \cdot L=L$.

Let us consider $V, L$. The functor $-L$ yielding a $\mathbb{C}$-linear combination of $V$ is defined as follows:

(Def. 10) $-L=\left(-1_{\mathbb{C}}\right) \cdot L$.

We now state three propositions:

(31) $(-L)(v)=-L(v)$.

(32) If $L_{1}+L_{2}=$ ZeroCLC $V$, then $L_{2}=-L_{1}$.

(33) $--L=L$.

Let us consider $V$ and let us consider $L_{1}, L_{2}$. The functor $L_{1}-L_{2}$ yields a $\mathbb{C}$-linear combination of $V$ and is defined by:

(Def. 11) $L_{1}-L_{2}=L_{1}+-L_{2}$.

One can prove the following propositions:

(34) $\left(L_{1}-L_{2}\right)(v)=L_{1}(v)-L_{2}(v)$.

(35) The support of $L_{1}-L_{2} \subseteq$ (the support of $\left.L_{1}\right) \cup$ (the support of $L_{2}$ ).

(36) Suppose $L_{1}$ is a $\mathbb{C}$-linear combination of $A$ and $L_{2}$ is a $\mathbb{C}$-linear combination of $A$. Then $L_{1}-L_{2}$ is a $\mathbb{C}$-linear combination of $A$.

(37) $L-L=$ ZeroCLC $V$. 
Let us consider $V$. The functor $\mathbb{C}$-LinComb $V$ yields a set and is defined as follows:

(Def. 12) $\quad x \in \mathbb{C}$-LinComb $V$ iff $x$ is a $\mathbb{C}$-linear combination of $V$.

Let us consider $V$. One can verify that $\mathbb{C}$-LinComb $V$ is non empty.

In the sequel $e, e_{1}, e_{2}$ denote elements of $\mathbb{C}$-LinComb $V$.

Let us consider $V$ and let us consider $e$. The functor ${ }^{\circledR} e$ yields a $\mathbb{C}$-linear combination of $V$ and is defined as follows:

(Def. 13) @ $e=e$.

Let us consider $V$ and let us consider $L$. The functor ${ }^{\circledR} L$ yielding an element of $\mathbb{C}$-LinComb $V$ is defined by:

(Def. 14) ${ }^{\circledR} L=L$.

Let us consider $V$. The functor $\mathbb{C}$-LCAdd $V$ yields a binary operation on $\mathbb{C}$-LinComb $V$ and is defined by:

(Def. 15) For all $e_{1}, e_{2}$ holds $(\mathbb{C}$-LCAdd $V)\left(e_{1}, e_{2}\right)=\left({ }^{@} e_{1}\right)+{ }^{\circledR} e_{2}$.

Let us consider $V$. The functor $\mathbb{C}$-LCMult $V$ yields a function from $\mathbb{C} \times$ $\mathbb{C}$-LinComb $V$ into $\mathbb{C}$-LinComb $V$ and is defined as follows:

(Def. 16) For all $a, e$ holds $(\mathbb{C}$-LCMult $V)(\langle a, e\rangle)=a \cdot\left({ }^{\circledR} e\right)$.

Let us consider $V$. The functor LCC-CLSpace $V$ yielding a complex linear space is defined by:

(Def. 17) LC-CLSpace $V=\left\langle\mathbb{C}\right.$-LinComb $V,{ }^{\circledR}$ ZeroCLC $V, \mathbb{C}$-LCAdd $V, \mathbb{C}$-LCMult $\left.V\right\rangle$.

Let us consider $V$. Note that LCC-CLSpace $V$ is strict and non empty.

We now state four propositions:

(38) $L_{1}{ }^{\mathrm{L} C \text {-CLSpace } V}+L_{2}{ }^{\mathrm{L}} \mathbb{C}$-CLSpace $V=L_{1}+L_{2}$.

(39) $a \cdot L^{\mathrm{L} C}$-CLSpace $V=a \cdot L$.

(40) $-L^{\mathrm{L} C-C L S p a c e} V=-L$.

(41) $L_{1}{ }^{\mathrm{L} C \text {-CLSpace } V}-L_{2}{ }^{\mathrm{L}} \mathbb{C}$-CLSpace $V=L_{1}-L_{2}$.

Let us consider $V$ and let us consider $A$. The functor LCC-CLSpace $A$ yielding a strict subspace of LC-CLSpace $V$ is defined as follows:

(Def. 18) The carrier of LC-CLSpace $A=\{l\}$.

\section{Preliminaries for Complex Convex Sets}

Let $V$ be a complex linear space and let $W$ be a subspace of $V$. The functor $\mathrm{Up}(W)$ yields a subset of $V$ and is defined by:

(Def. 19) $\quad \mathrm{Up}(W)=$ the carrier of $W$.

Let $V$ be a complex linear space and let $W$ be a subspace of $V$. One can check that $\mathrm{Up}(W)$ is non empty. 
Let $V$ be a non empty CLS structure and let $S$ be a subset of $V$. We say that $S$ is affine if and only if the condition (Def. 20) is satisfied.

(Def. 20) Let $x, y$ be vectors of $V$ and $z$ be a complex number. If there exists a real number $a$ such that $a=z$ and $x, y \in S$, then $\left(1_{\mathbb{C}}-z\right) \cdot x+z \cdot y \in S$.

Let $V$ be a complex linear space. The functor $\Omega_{V}$ yields a strict subspace of $V$ and is defined as follows:

(Def. 21) $\Omega_{V}=$ the CLS structure of $V$.

Let $V$ be a non empty CLS structure. Observe that $\Omega_{V}$ is affine and $\emptyset_{V}$ is affine.

Let $V$ be a non empty CLS structure. One can check that there exists a subset of $V$ which is non empty and affine and there exists a subset of $V$ which is empty and affine.

We now state three propositions:

(42) For every real number $a$ and for every complex number $z$ holds $\Re(a \cdot z)=$ $a \cdot \Re(z)$.

(43) For every real number $a$ and for every complex number $z$ holds $\Im(a \cdot z)=$ $a \cdot \Im(z)$.

(44) For every real number $a$ and for every complex number $z$ such that $0 \leq a \leq 1$ holds $|a \cdot z|=a \cdot|z|$ and $\left|\left(1_{\mathbb{C}}-a\right) \cdot z\right|=\left(1_{\mathbb{C}}-a\right) \cdot|z|$.

\section{Complex Convex Sets}

Let $V$ be a non empty CLS structure, let $M$ be a subset of $V$, and let $r$ be an element of $\mathbb{C}$. The functor $r \cdot M$ yielding a subset of $V$ is defined by:

(Def. 22) $\quad r \cdot M=\{r \cdot v ; v$ ranges over elements of $V: v \in M\}$.

Let $V$ be a non empty CLS structure and let $M$ be a subset of $V$. We say that $M$ is convex if and only if the condition (Def. 23) is satisfied.

(Def. 23) Let $u, v$ be vectors of $V$ and $z$ be a complex number. Suppose there exists a real number $r$ such that $z=r$ and $0<r<1$ and $u, v \in M$. Then $z \cdot u+\left(1_{\mathbb{C}}-z\right) \cdot v \in M$.

One can prove the following propositions:

(45) Let $V$ be a complex linear space-like non empty CLS structure, $M$ be a subset of $V$, and $z$ be a complex number. If $M$ is convex, then $z \cdot M$ is convex.

(46) Let $V$ be an Abelian add-associative complex linear space-like non empty CLS structure and $M, N$ be subsets of $V$. If $M$ is convex and $N$ is convex, then $M+N$ is convex.

(47) Let $V$ be a complex linear space and $M, N$ be subsets of $V$. If $M$ is convex and $N$ is convex, then $M-N$ is convex. 
(48) Let $V$ be a non empty CLS structure and $M$ be a subset of $V$. Then $M$ is convex if and only if for every complex number $z$ such that there exists a real number $r$ such that $z=r$ and $0<r<1$ holds $z \cdot M+\left(1_{\mathbb{C}}-z\right) \cdot M \subseteq M$.

(49) Let $V$ be an Abelian non empty CLS structure and $M$ be a subset of $V$. Suppose $M$ is convex. Let $z$ be a complex number. If there exists a real number $r$ such that $z=r$ and $0<r<1$, then $\left(1_{\mathbb{C}}-z\right) \cdot M+z \cdot M \subseteq M$.

(50) Let $V$ be an Abelian add-associative complex linear space-like non empty CLS structure and $M, N$ be subsets of $V$. Suppose $M$ is convex and $N$ is convex. Let $z$ be a complex number. If there exists a real number $r$ such that $z=r$, then $z \cdot M+\left(1_{\mathbb{C}}-z\right) \cdot N$ is convex.

(51) For every complex linear space-like non empty CLS structure $V$ and for every subset $M$ of $V$ holds $1_{\mathbb{C}} \cdot M=M$.

(52) For every complex linear space $V$ and for every non empty subset $M$ of $V$ holds $0_{\mathbb{C}} \cdot M=\left\{0_{V}\right\}$.

(53) For every add-associative non empty additive loop structure $V$ and for all subsets $M_{1}, M_{2}, M_{3}$ of $V$ holds $\left(M_{1}+M_{2}\right)+M_{3}=M_{1}+\left(M_{2}+M_{3}\right)$.

(54) Let $V$ be a complex linear space-like non empty CLS structure, $M$ be a subset of $V$, and $z_{1}, z_{2}$ be complex numbers. Then $z_{1} \cdot\left(z_{2} \cdot M\right)=\left(z_{1} \cdot z_{2}\right) \cdot M$.

(55) Let $V$ be a complex linear space-like non empty CLS structure, $M_{1}$, $M_{2}$ be subsets of $V$, and $z$ be a complex number. Then $z \cdot\left(M_{1}+M_{2}\right)=$ $z \cdot M_{1}+z \cdot M_{2}$.

(56) Let $V$ be a complex linear space, $M$ be a subset of $V$, and $v$ be a vector of $V$. Then $M$ is convex if and only if $v+M$ is convex.

(57) For every complex linear space $V$ holds $\mathrm{Up}\left(\mathbf{0}_{V}\right)$ is convex.

(58) For every complex linear space $V$ holds $\operatorname{Up}\left(\Omega_{V}\right)$ is convex.

(59) For every non empty CLS structure $V$ and for every subset $M$ of $V$ such that $M=\emptyset$ holds $M$ is convex.

(60) Let $V$ be an Abelian add-associative complex linear space-like non empty CLS structure, $M_{1}, M_{2}$ be subsets of $V$, and $z_{1}, z_{2}$ be complex numbers. If $M_{1}$ is convex and $M_{2}$ is convex, then $z_{1} \cdot M_{1}+z_{2} \cdot M_{2}$ is convex.

(61) Let $V$ be a complex linear space-like non empty CLS structure, $M$ be a subset of $V$, and $z_{1}, z_{2}$ be complex numbers. Then $\left(z_{1}+z_{2}\right) \cdot M \subseteq$ $z_{1} \cdot M+z_{2} \cdot M$.

(62) Let $V$ be a non empty CLS structure, $M, N$ be subsets of $V$, and $z$ be a complex number. If $M \subseteq N$, then $z \cdot M \subseteq z \cdot N$.

(63) For every non empty CLS structure $V$ and for every empty subset $M$ of $V$ and for every complex number $z$ holds $z \cdot M=\emptyset$.

(64) Let $V$ be a non empty additive loop structure, $M$ be an empty subset of $V$, and $N$ be a subset of $V$. Then $M+N=\emptyset$. 
(65) For every right zeroed non empty additive loop structure $V$ and for every subset $M$ of $V$ holds $M+\left\{0_{V}\right\}=M$.

(66) Let $V$ be a complex linear space, $M$ be a subset of $V$, and $z_{1}, z_{2}$ be complex numbers. Suppose there exist real numbers $r_{1}, r_{2}$ such that $z_{1}=$ $r_{1}$ and $z_{2}=r_{2}$ and $r_{1} \geq 0$ and $r_{2} \geq 0$ and $M$ is convex. Then $z_{1} \cdot M+z_{2}$. $M=\left(z_{1}+z_{2}\right) \cdot M$.

(67) Let $V$ be an Abelian add-associative complex linear space-like non empty CLS structure, $M_{1}, M_{2}, M_{3}$ be subsets of $V$, and $z_{1}, z_{2}, z_{3}$ be complex numbers. If $M_{1}$ is convex and $M_{2}$ is convex and $M_{3}$ is convex, then $z_{1}$. $M_{1}+z_{2} \cdot M_{2}+z_{3} \cdot M_{3}$ is convex.

(68) Let $V$ be a non empty CLS structure and $F$ be a family of subsets of $V$. Suppose that for every subset $M$ of $V$ such that $M \in F$ holds $M$ is convex. Then $\bigcap F$ is convex.

(69) For every non empty CLS structure $V$ and for every subset $M$ of $V$ such that $M$ is affine holds $M$ is convex.

Let $V$ be a non empty CLS structure. One can check that there exists a subset of $V$ which is non empty and convex.

Let $V$ be a non empty CLS structure. Observe that there exists a subset of $V$ which is empty and convex.

One can prove the following propositions:

(70) Let $V$ be a complex unitary space-like non empty complex unitary space structure, $M$ be a subset of $V, v$ be a vector of $V$, and $r$ be a real number. If $M=\{u ; u$ ranges over vectors of $V: \Re((u \mid v)) \geq r\}$, then $M$ is convex.

(71) Let $V$ be a complex unitary space-like non empty complex unitary space structure, $M$ be a subset of $V, v$ be a vector of $V$, and $r$ be a real number. If $M=\{u ; u$ ranges over vectors of $V: \Re((u \mid v))>r\}$, then $M$ is convex.

(72) Let $V$ be a complex unitary space-like non empty complex unitary space structure, $M$ be a subset of $V, v$ be a vector of $V$, and $r$ be a real number. If $M=\{u ; u$ ranges over vectors of $V: \Re((u \mid v)) \leq r\}$, then $M$ is convex.

(73) Let $V$ be a complex unitary space-like non empty complex unitary space structure, $M$ be a subset of $V, v$ be a vector of $V$, and $r$ be a real number. If $M=\{u ; u$ ranges over vectors of $V: \Re((u \mid v))<r\}$, then $M$ is convex.

(74) Let $V$ be a complex unitary space-like non empty complex unitary space structure, $M$ be a subset of $V, v$ be a vector of $V$, and $r$ be a real number. If $M=\{u ; u$ ranges over vectors of $V: \Im((u \mid v)) \geq r\}$, then $M$ is convex.

(75) Let $V$ be a complex unitary space-like non empty complex unitary space structure, $M$ be a subset of $V, v$ be a vector of $V$, and $r$ be a real number. If $M=\{u ; u$ ranges over vectors of $V: \Im((u \mid v))>r\}$, then $M$ is convex.

(76) Let $V$ be a complex unitary space-like non empty complex unitary space structure, $M$ be a subset of $V, v$ be a vector of $V$, and $r$ be a real number. 
If $M=\{u ; u$ ranges over vectors of $V: \Im((u \mid v)) \leq r\}$, then $M$ is convex.

(77) Let $V$ be a complex unitary space-like non empty complex unitary space structure, $M$ be a subset of $V, v$ be a vector of $V$, and $r$ be a real number. If $M=\{u ; u$ ranges over vectors of $V: \Im((u \mid v))<r\}$, then $M$ is convex.

(78) Let $V$ be a complex unitary space-like non empty complex unitary space structure, $M$ be a subset of $V, v$ be a vector of $V$, and $r$ be a real number. If $M=\{u ; u$ ranges over vectors of $V:|(u \mid v)| \leq r\}$, then $M$ is convex.

(79) Let $V$ be a complex unitary space-like non empty complex unitary space structure, $M$ be a subset of $V, v$ be a vector of $V$, and $r$ be a real number. If $M=\{u ; u$ ranges over vectors of $V:|(u \mid v)|<r\}$, then $M$ is convex.

\section{Complex Convex Combinations}

Let $V$ be a complex linear space and let $L$ be a $\mathbb{C}$-linear combination of $V$. We say that $L$ is convex if and only if the condition (Def. 24) is satisfied.

(Def. 24) There exists a finite sequence $F$ of elements of the carrier of $V$ such that

(i) $F$ is one-to-one,

(ii) $\operatorname{rng} F=$ the support of $L$, and

(iii) there exists a finite sequence $f$ of elements of $\mathbb{R}$ such that len $f=\operatorname{len} F$ and $\sum f=1$ and for every natural number $n$ such that $n \in \operatorname{dom} f$ holds $f(n)=L(F(n))$ and $f(n) \geq 0$.

We now state several propositions:

(80) Let $V$ be a complex linear space and $L$ be a $\mathbb{C}$-linear combination of $V$. If $L$ is convex, then the support of $L \neq \emptyset$.

(81) Let $V$ be a complex linear space, $L$ be a $\mathbb{C}$-linear combination of $V$, and $v$ be a vector of $V$. Suppose $L$ is convex and there exists a real number $r$ such that $r=L(v)$ and $r \leq 0$. Then $v \notin$ the support of $L$.

(82) For every complex linear space $V$ and for every $\mathbb{C}$-linear combination $L$ of $V$ such that $L$ is convex holds $L \neq$ ZeroCLC $V$.

(83) Let $V$ be a complex linear space, $v$ be a vector of $V$, and $L$ be a $\mathbb{C}$ linear combination of $V$. Suppose $L$ is convex and the support of $L=\{v\}$. Then there exists a real number $r$ such that $r=L(v)$ and $r=1$ and $\sum L=L(v) \cdot v$.

(84) Let $V$ be a complex linear space, $v_{1}, v_{2}$ be vectors of $V$, and $L$ be a $\mathbb{C}$-linear combination of $V$. Suppose $L$ is convex and the support of $L=\left\{v_{1}, v_{2}\right\}$ and $v_{1} \neq v_{2}$. Then there exist real numbers $r_{1}, r_{2}$ such that $r_{1}=L\left(v_{1}\right)$ and $r_{2}=L\left(v_{2}\right)$ and $r_{1}+r_{2}=1$ and $r_{1} \geq 0$ and $r_{2} \geq 0$ and $\sum L=L\left(v_{1}\right) \cdot v_{1}+L\left(v_{2}\right) \cdot v_{2}$. 
(85) Let $V$ be a complex linear space, $v_{1}, v_{2}, v_{3}$ be vectors of $V$, and $L$ be a $\mathbb{C}$-linear combination of $V$. Suppose $L$ is convex and the support of $L=\left\{v_{1}, v_{2}, v_{3}\right\}$ and $v_{1} \neq v_{2} \neq v_{3} \neq v_{1}$. Then

(i) there exist real numbers $r_{1}, r_{2}, r_{3}$ such that $r_{1}=L\left(v_{1}\right)$ and $r_{2}=L\left(v_{2}\right)$ and $r_{3}=L\left(v_{3}\right)$ and $r_{1}+r_{2}+r_{3}=1$ and $r_{1} \geq 0$ and $r_{2} \geq 0$ and $r_{3} \geq 0$, and

(ii) $\sum L=L\left(v_{1}\right) \cdot v_{1}+L\left(v_{2}\right) \cdot v_{2}+L\left(v_{3}\right) \cdot v_{3}$.

(86) Let $V$ be a complex linear space, $v$ be a vector of $V$, and $L$ be a $\mathbb{C}$-linear combination of $\{v\}$. Suppose $L$ is convex. Then there exists a real number $r$ such that $r=L(v)$ and $r=1$ and $\sum L=L(v) \cdot v$.

(87) Let $V$ be a complex linear space, $v_{1}, v_{2}$ be vectors of $V$, and $L$ be a $\mathbb{C}$-linear combination of $\left\{v_{1}, v_{2}\right\}$. Suppose $v_{1} \neq v_{2}$ and $L$ is convex. Then there exist real numbers $r_{1}, r_{2}$ such that $r_{1}=L\left(v_{1}\right)$ and $r_{2}=L\left(v_{2}\right)$ and $r_{1} \geq 0$ and $r_{2} \geq 0$ and $\sum L=L\left(v_{1}\right) \cdot v_{1}+L\left(v_{2}\right) \cdot v_{2}$.

(88) Let $V$ be a complex linear space, $v_{1}, v_{2}, v_{3}$ be vectors of $V$, and $L$ be a $\mathbb{C}$-linear combination of $\left\{v_{1}, v_{2}, v_{3}\right\}$. Suppose $v_{1} \neq v_{2} \neq v_{3} \neq v_{1}$ and $L$ is convex. Then

(i) there exist real numbers $r_{1}, r_{2}, r_{3}$ such that $r_{1}=L\left(v_{1}\right)$ and $r_{2}=L\left(v_{2}\right)$ and $r_{3}=L\left(v_{3}\right)$ and $r_{1}+r_{2}+r_{3}=1$ and $r_{1} \geq 0$ and $r_{2} \geq 0$ and $r_{3} \geq 0$, and

(ii) $\quad \sum L=L\left(v_{1}\right) \cdot v_{1}+L\left(v_{2}\right) \cdot v_{2}+L\left(v_{3}\right) \cdot v_{3}$.

\section{Complex Convex Hull}

Let $V$ be a non empty CLS structure and let $M$ be a subset of $V$. The functor Convex-Family $M$ yielding a family of subsets of $V$ is defined by:

(Def. 25) For every subset $N$ of $V$ holds $N \in$ Convex-Family $M$ iff $N$ is convex and $M \subseteq N$.

Let $V$ be a non empty CLS structure and let $M$ be a subset of $V$. The functor conv $M$ yielding a convex subset of $V$ is defined as follows:

(Def. 26) $\operatorname{conv} M=\bigcap$ Convex-Family $M$.

The following proposition is true

(89) Let $V$ be a non empty CLS structure, $M$ be a subset of $V$, and $N$ be a convex subset of $V$. If $M \subseteq N$, then conv $M \subseteq N$.

\section{REFERENCES}

[1] Grzegorz Bancerek. Cardinal numbers. Formalized Mathematics, 1(2):377-382, 1990.

[2] Grzegorz Bancerek. The ordinal numbers. Formalized Mathematics, 1(1):91-96, 1990.

[3] Grzegorz Bancerek and Krzysztof Hryniewiecki. Segments of natural numbers and finite sequences. Formalized Mathematics, 1(1):107-114, 1990.

[4] Czesław Byliński. Binary operations. Formalized Mathematics, 1(1):175-180, 1990. 
[5] Czesław Byliński. The complex numbers. Formalized Mathematics, 1(3):507-513, 1990.

[6] Czesław Byliński. Functions and their basic properties. Formalized Mathematics, 1(1):55$65,1990$.

[7] Czesław Byliński. Functions from a set to a set. Formalized Mathematics, 1(1):153-164, 1990.

[8] Czesław Byliński. Partial functions. Formalized Mathematics, 1(2):357-367, 1990.

[9] Czesław Byliński. Some basic properties of sets. Formalized Mathematics, 1(1):47-53, 1990.

[10] Czesław Byliński. The sum and product of finite sequences of real numbers. Formalized Mathematics, 1(4):661-668, 1990.

[11] Agata Darmochwał. Finite sets. Formalized Mathematics, 1(1):165-167, 1990.

[12] Noboru Endou. Complex linear space and complex normed space. Formalized Mathematics, 12(2):93-102, 2004.

[13] Noboru Endou. Complex linear space of complex sequences. Formalized Mathematics, 12(2):109-117, 2004.

[14] Noboru Endou, Takashi Mitsuishi, and Yasunari Shidama. Dimension of real unitary space. Formalized Mathematics, 11(1):23-28, 2003.

[15] Noboru Endou, Takashi Mitsuishi, and Yasunari Shidama. Topology of real unitary space. Formalized Mathematics, 11(1):33-38, 2003.

[16] Krzysztof Hryniewiecki. Basic properties of real numbers. Formalized Mathematics, 1(1):35-40, 1990.

[17] Beata Padlewska. Families of sets. Formalized Mathematics, 1(1):147-152, 1990.

[18] Andrzej Trybulec. Domains and their Cartesian products. Formalized Mathematics, 1(1):115-122, 1990.

[19] Andrzej Trybulec. Enumerated sets. Formalized Mathematics, 1(1):25-34, 1990.

[20] Andrzej Trybulec. Function domains and Frænkel operator. Formalized Mathematics, 1(3):495-500, 1990.

[21] Wojciech A. Trybulec. Linear combinations in real linear space. Formalized Mathematics, 1(3):581-588, 1990.

[22] Wojciech A. Trybulec. Vectors in real linear space. Formalized Mathematics, 1(2):291-296, 1990.

[23] Zinaida Trybulec. Properties of subsets. Formalized Mathematics, 1(1):67-71, 1990.

[24] Edmund Woronowicz. Relations and their basic properties. Formalized Mathematics, 1(1):73-83, 1990

[25] Edmund Woronowicz. Relations defined on sets. Formalized Mathematics, 1(1):181-186, 1990. 